\title{
MULTI-VALUED MAPPINGS AND FIXED POINTS II
}

\author{
B. C. DHAGE
}

\begin{abstract}
In this paper, some hybrid fixed point principles for the sum of two multi-valued operators in a Banach space are proved and they are further applied to a certain integral inclusion of mixed type for proving the existence results under mixed Lipschitz and Carathéodory conditions.
\end{abstract}

\section{Introduction}

Hybrid fixed point theorem of Krasnoselskii [12] which combines a topological fixed point theorem of Schauder with a geometrical fixed point theorem of Banach has been a subject of great interest for a long time. It is known that the fixed point theorem of Krasnoselskii has nice applications to perturbed nonlinear differential and the mixed type of integral equations including the allied areas of mathematics for proving the existence theorems under mixed Lipschitz and compactness conditions. See [6], [7], [16], $[19],[23]$ and the references therein. There are several extensions and generalizations of Krasnoselskii fixed point theorem in the course of time. Recently Petruşel [16] has obtained a multi-valued analogue of this theorem under some relaxed conditions and which is further applied to a certain integral inclusion for proving the existence of solutions. In this paper, we prove some nonlinear alternatives of Leray-Schauder type for the multivalued version of Krasnoselskii fixed point theorem involving the sum of two multi-valued operators in a Banach space and apply them to prove the existence results for a certain functional integral inclusion under mixed Lipschitz and Carathéodory conditions.

\section{Preliminaries}

Throughout this paper, unless otherwise mentioned, let $X$ denote a Banach space with norm $\|\cdot\|$. Let $\mathcal{P}_{p}(X)$ denote the class of all non-empty subsets of $X$ with property $p$. In particular, $\mathcal{P}_{b d, c l}(X)$ and $\mathcal{P}_{c p, c v}(X)$ denote respectively the classes of all bounded and closed, and compact and convex subsets of $X$. For $x \in X$ and $Y, Z \in \mathcal{P}_{b d, c l}(X)$, we denote $D(x, Y)=\inf \{\|x-y\| \mid y \in Y\}$, and $\rho(Y, Z)=\sup _{a \in Y} D(a, Z)$. Define a function $H: \mathcal{P}_{c l}(X) \times \mathcal{P}_{c l}(X) \rightarrow \mathbb{R}^{+}$by

$$
H(A, B)=\max \{\rho(A, B), \rho(B, A)\} .
$$

Received and revised July 27, 2004.

2000 Mathematics Subject Classification. 47H10.

Key words and phrases. Multi-valued map, fixed point theorem and differential inclusion. 
Note that $\|Y\|=H(Y,\{0\})$.

A correspondence $T: X \rightarrow \mathcal{P}_{p}(X)$ is called a multi-valued mapping on $X$. A point $x_{0} \in X$ is called a fixed point of the multi-valued operator $T: X \rightarrow \mathcal{P}_{p}(X)$ if $x_{0} \in T\left(x_{0}\right)$. The fixed points set of $T$ will be denoted by $F i x(T)$.

Definition 2.1. Let $T: X \rightarrow \mathcal{P}_{b d, c l}(X)$ be a multi-valued operator. Then $T$ is called a multi-valued contraction on $X$ if there exists a constant $k \in(0,1)$ such that

$$
H(T(x), T(y)) \leq k\|x-y\|
$$

for all $x, y \in X$. The constant $k$ is called a contraction constant of $T$.

The following fixed point theorem for multi-valued contraction mappings appears in Covitz and Nadler [4].

Theorem 2.1. Let $(X, d)$ be a complete metric space and let $T: X \rightarrow \mathcal{P}_{c l}(X)$ be a multi-valued contraction. Then Fix $(T)$ is a non-empty and closed subset of $X$.

Let $X$ be a metric space. A multi-valued mapping $T: X \rightarrow \mathcal{P}_{p}(X)$ is called lower semi-continuous (resp. an upper semi-continuous) if $G$ is any open subset of $X$, then $\{x \in X \mid T x \cap G \neq \emptyset\}$ (resp. $\{x \in X \mid T x \subset G\})$ is an open subset of $X$. The multivalued operator $T$ is called totally compact if $\overline{T(S)}$ is a compact subset of $X$ for any $S \subset X . T$ is called compact if $\overline{T(S)}$ is a relatively compact subset of $X$ for all bounded subsets $S$ of $X$. Again $T$ is called totally bounded if for any bounded subset $S$ of $X, T(S)$ is a totally bounded subset of $X$. A multi-valued operator $T: X \rightarrow \mathcal{P}_{p}(X)$ is called completely continuous if it is upper semi-continuous and compact on $X$. Every compact multi-valued operator is totally bounded but the converse may not be true. However, these two notions are equivalent on a bounded subset in a complete metric space $X$.

The Leray-Schauder nonlinear alternative for compact single-valued mappings is

Theorem 2.2. Let $U$ and $\bar{U}$ be open and closed subsets of a Banach space $X$ respectively such that $0 \in U$ and let $T: \bar{U} \rightarrow X$ be continuous and totally compact mapping. Then either

(i) T has a fixed point, or

(ii) there exists an element $u \in \partial U$ such that $\lambda u=T u$ for some $\lambda>1$, where $\partial U$ is the boundary of $U$.

The Hausdorff measure of noncompactness for a bounded subset $S$ of $X$ is a nonnegative real number $\chi(S)$ defined by

$$
\chi(S)=\inf \left\{r>0: S \subset \bigcup_{i=1}^{n} \mathcal{B}_{i}\left(x_{i}, r\right), \text { for some } x_{i} \in X\right\},
$$

where $\mathcal{B}_{i}\left(x_{i}, r\right)=\left\{x \in X \mid d\left(x, x_{i}\right)<r\right\}$.

The measure of noncompactness $\chi$ enjoys the following properties: 
$\left(\chi_{1}\right) \chi(A)=0 \Leftrightarrow A$ is relatively compact.

$\left(\chi_{2}\right) \chi(A)=\chi(\bar{A})=\chi(\overline{c o} A)$, where $\bar{A}$ and $\overline{c o} A$ denote respectively the closure and the closed convex hull of $A$.

$\left(\chi_{3}\right) A \subset B \Rightarrow \chi(A) \leq \alpha(B)$.

$\left(\chi_{4}\right) \chi(A \cup B)=\max \{\chi(A), \chi(B)\}$.

$\left(\chi_{5}\right) \chi(\lambda A)=|\lambda| \chi(A), \forall \lambda \in \mathbb{R}$.

( $\left.\chi_{6}\right) \chi(A+B) \leq \chi(A)+\chi(B)$.

The details of Hausdorff measure of noncompactness and its properties appear in Deimling [5], Zeidler [24] and the references therein.

Definition 2.2. A multi-valued mapping $T: X \rightarrow \mathcal{P}_{c p}(X)$ is called condensing if for any bounded subset $S$ of $X, T(S)$ is bounded and $\chi(T(S))<\chi(S)$ for $\chi(S)>0$.

It is known that contraction and completely continuous mappings are condensing, but the converse may not be true. See Petruşel $[16,17]$. The one of the famous fixed point theorems for condensing multi-valued mappings is the following variant of a fixed point theorem of O'Regan [20] under slightly weaker condition.

Theorem 2.3. Let $U$ and $\bar{U}$ be open and closed subsets of a Banach space $X$ respectively such that $0 \in U$ and let $T: \bar{U} \rightarrow \mathcal{P}_{c p, c v}(X)$ be an upper semi-continuous and condensing mapping such that $T(\bar{U})$ is bounded. Then either

(i) Thas a fixed point, or

(ii) there exists an element $u \in \partial U$ such that $\lambda u \in T u$ for some $\lambda>1$, where $\partial U$ is the boundary of $U$.

In the following section we prove the main fixed point theorems of this paper.

\section{Multi-valued Fixed Point Theory}

Before going to the main fixed point results of this section, we state some of the results which are useful in the sequel.

Theorem 3.1.(Rybinski [22]) Let $S$ be a nonempty and closed subset of a Banach space $X$ and let $Y$ be a metric space. Assume that $F: S \times Y \rightarrow \mathcal{P}_{c l, c v}(S)$ be a multi-valued mapping satisfying

(a) $H\left(F\left(x_{1}, y\right), F\left(x_{2}, y\right)\right) \leq k\left\|x_{1}-x_{2}\right\|$ for each $\left(x_{1}, y\right),\left(x_{2}, y\right) \in S \times Y$,

(b) for every $x \in S, F(x, \cdot)$ is lower semi-continuous (briefly l.s.c.) on $Y$.

Then there exists a continuous mapping $f: S \times Y \rightarrow S$ such that $f(x, y) \in F(f(x, y), y)$ for each $(x, y) \in S \times Y$.

The following lemmas are useful in the sequel.

Lemma 3.1.([Lim [14]) Let $(X, d)$ be a complete metric space and let $T_{1}, T_{2}: X \rightarrow$ 
$\mathcal{P}_{b d, c l}(X)$ be two multi-valued contractions with the same contraction constant $k$. Then

$$
\rho\left(F i x\left(T_{1}\right), F i x\left(T_{2}\right)\right) \leq \frac{1}{1-k} \sup _{x \in X} \rho\left(T_{1}(x), T_{2}(x)\right) .
$$

Lemma 3.2. (Hu and Papageorgiou [10]) Let $X$ be a Banach space. Then

$$
H(Y+Z, Y+W) \leq H(Z, W)
$$

for $Y, Z, W \in \mathcal{P}_{b d}(X)$.

Lemma 3.3. (Kisielewicz [10]) Let $X$ be a Banach space. Then

$$
H(\lambda Y, \lambda Z) \leq|\lambda| H(Z, W)
$$

for $Y, Z \in \mathcal{P}_{b d}(X)$ and for each $\lambda \in \mathbb{R}$.

Theorem 3.2. Let $U$ be an open subset of $X$ such that $0 \in U$ and let $A: X \rightarrow$ $\mathcal{P}_{b d, c l, c v}(X), B: \bar{U} \rightarrow \mathcal{P}_{c p, c v}(X)$ be two multi-valued operators satisfying

(a) $A$ is contraction with a contraction constant $k$, and

(b) B is l.s.c. and totally compact.

Then either

(i) the operator inclusion $\lambda x \in A(\lambda x)+B x$ has a solution in $\bar{U}$ for $\lambda=1$, or

(ii) there exists an element $u \in \partial U$ such that $\lambda x \in A(\lambda x)+B x$ for some $\lambda>1$, where $\partial U$ is the boundary of $U$.

Proof. Define a multi-valued operator $T: X \times \bar{U} \rightarrow \mathcal{P}_{c l, c v, b d}(X)$ by

$$
T(x, y)=A x+B y .
$$

We show that $T(x, y)$ is multi-valued contraction operator in $x$ for each fixed $y \in X$. Let $x_{1}, x_{2} \in X$ be arbitrary. Then by Lemma 3.2,

$$
\begin{aligned}
H\left(T\left(x_{1}, y\right), T\left(x_{2}, y\right)\right) & =H\left(A\left(x_{1}\right)+B(y), A\left(x_{2}\right)+B(y)\right) \\
& \leq H\left(A\left(x_{1}\right), A\left(x_{2}\right)\right) \\
& \leq k\left\|x_{1}-x_{2}\right\| .
\end{aligned}
$$

This shows that the multi-valued operator $T_{y}(\cdot)=T(., y)$ is a contraction on $\mathrm{X}$ with a contraction constant $k$. Hence an application of Covitz-Nadler fixed point theorem yields that the fixed point set

$$
\operatorname{Fix}\left(T_{y}\right)=\{x \in X \mid x \in A(x)+B(y)\}
$$

is nonempty and closed for each $y \in X$.

Now the operator $T(x, y)$ satisfies all the conditions of Theorem 3.1 and hence there exists a continuous mapping $f: X \times \bar{U} \rightarrow X$ such that $f(x, y) \in A(f(x, y))+B(y)$. Let 
us define $C(y)=F i x\left(T_{y}\right), C: \bar{U} \rightarrow \mathcal{P}_{c l}(X)$. Let us consider the single-valued operator $c: \bar{U} \rightarrow X$ defined by $c(x)=f(x, x)$, for each $x \in \bar{U}$. Then $c$ is a continuous mapping having the property that

$$
c(x)=f(x, x) \in A(f(x, x))+B(x)=A(c(x))+B(x),
$$

for each $x \in \bar{U}$.

Now, we will prove that $c$ is compact on $\bar{U}$. To do this, it is sufficient to show that $C$ is compact on $\bar{U}$. Let $\epsilon>0$. Since $B$ is totally compact on $\bar{U}, B(\bar{U})$ is compact. Then there exists $Y=\left\{y_{1}, \ldots, y_{n}\right\} \subset X$ such that

$$
\begin{aligned}
B(\bar{U}) & \subset\left\{w_{1}, \ldots, w_{n}\right\}+\mathcal{B}(0,(1-k) \epsilon) \\
& \subset \bigcup_{i=1}^{n} B\left(y_{i}\right)+\mathcal{B}(0,(1-k) \epsilon),
\end{aligned}
$$

where $w_{i} \in B\left(y_{i}\right)$, for each $i=1,2, \ldots, n$. It follows that, for each $y \in \bar{U}$,

$$
B(y) \subset \bigcup_{i=1}^{n} B\left(y_{i}\right)+\mathcal{B}(0,(1-k) \epsilon)
$$

and hence there exists an element $y_{k} \in Y$ such that

$$
\rho\left(B(y), B\left(y_{k}\right)\right)<(1-k) \epsilon .
$$

Then

$$
\begin{aligned}
\rho\left(C(y), C\left(y_{k}\right)\right) & =\rho\left(\operatorname{Fix}\left(T_{y}\right), \operatorname{Fix}\left(T_{y_{k}}\right)\right) \\
& \leq \frac{1}{1-k} \sup _{x \in \bar{U}} \rho\left(T_{y}(x), T_{y_{k}}(x)\right) \\
& =\frac{1}{1-k} \sup _{x \in \bar{U}} \rho\left(A(x)+B(y), A(x)+B\left(y_{k}\right)\right) \\
& \leq \frac{1}{1-k} \sup _{x \in \bar{U}} \rho\left(B(y), B\left(y_{k}\right)\right) \\
& <\frac{1}{1-k}(1-k) \epsilon \\
& =\epsilon .
\end{aligned}
$$

It follows that for each $u \in C(y)$ there is $v_{k} \in C\left(y_{k}\right)$ such that $\left\|u-v_{k}\right\|<\epsilon$. Hence, for each $y \in \bar{U}, C(y) \subset \bigcup_{i=1}^{n} \mathcal{B}_{\epsilon}\left(v_{i}\right)$, where $v_{i} \in C\left(y_{i}\right), i=1,2, \ldots, n$. Therefore $c(\bar{U}) \subset C(\bar{U}) \subset \bigcup_{i=1}^{n} \mathcal{B}_{\epsilon}\left(v_{i}\right)$ and so $c$ is a totally compact operator on $\bar{U}$.

Finally, note that the mapping $c: \bar{U} \rightarrow X$ satisfies all the assumptions of LeraySchauder's nonlinear alternative and hence an application of it yields that either 
(i) the operator equation $\lambda x=c(x) \in A(c(x))+B x=A(\lambda x)+B x$ has a solution in $\bar{U}$ for $\lambda=1$, or

(ii) there exists an element $u \in \partial U$ such that $\lambda u=c(u) \in A(c(u))+B x=A(\lambda u)+B u$ for some $\lambda>1$, where $\partial U$ is the boundary of $U$.

This completes the proof.

Corollary 3.1. Let $\mathcal{B}_{r}(0)$ and $\overline{\mathcal{B}_{r}(0)}$ denote respectively the open and closed balls in a Banach space $X$ centered at origin 0 of radius $r$. Let $A: X \rightarrow \mathcal{P}_{c l, c v, b d}(X)$, $B: \overline{\mathcal{B}_{r}(0)} \rightarrow \mathcal{P}_{c p, c v}(X)$ be two multi-valued operators satisfying

(a) $A$ is contraction, and

(b) B is l.s.c. and totally compact.

Then either

(i) the operator inclusion $\lambda x \in A(\lambda x)+B x$ has a solution in $\overline{\mathcal{B}_{r}(0)}$ for $\lambda=1$, or

(ii) there exists an element $X$ with $\|u\|=r$ satisfying $\lambda u \in A(\lambda u)+B u$ for some $\lambda>1$, where $\partial U$ is a boundary of $U$.

To prove the next fixed point theorem, we need the following result of Petryshyn and Fitzpatrik [18] in the sequel.

Lemma 3.4. Let Let $X$ be a metric space and let $A: X \rightarrow \mathcal{P}_{b d, c l}(X)$ be a multi-valued contraction with a contraction $k$. Then for any bounded subset $S$ of $X, \chi(A(S)) \leq k \chi(S)$.

Theorem 3.3. Let $U$ and $\bar{U}$ be respectively the open and closed subsets of a Banach space $X$ such that $0 \in U$. Let $A: \bar{U} \rightarrow \mathcal{P}_{c l, c v, b d}(X)$ and $B: \bar{U} \rightarrow \mathcal{P}_{c p, c v}(X)$ be two multi-valued operators such that $A(\bar{U})+B(\bar{U})$ is bounded. Suppose that

(a) $A$ is contraction with a contraction $k$,

(b) B is u.s.c. and compact.

Then either

(i) the operator inclusion $\lambda x \in A x+B x$ has a solution for $\lambda=1$, or

(ii) there is an element $u \in \partial U$ such that $\lambda u \in A u+B u$ for some $\lambda>1$, where $\partial U$ is the boundary of $U$.

Proof. Since $A$ is multi-valued contraction, it is both lower as well as upper semicontinuous on $X$. Therefore the multi-valued operator $T: X \rightarrow X$ defined by $T(x)=$ $(A+B)(x)=A x+B x$ is upper semi-continuous on $X$. Obviously $A: \bar{U} \rightarrow \mathcal{P}_{c p, c v}(X)$, since $\alpha(A x) \leq k \alpha(\{x\})=0$. As a result, we have $T: \bar{U} \rightarrow \mathcal{P}_{c p, c v}(X)$. Let $S$ be a bounded subset of $\bar{U}$. Then by property $\left(\chi_{6}\right)$,

$$
\chi(T(S)) \leq \chi(A(S)+B(S)) \leq \chi(A(S))+\chi(B(S)) .
$$

As $B$ is completely continuous, we have that $\chi(B(S))=0$. Again from Lemma 3.3, it follows that $\chi(A(S)) \leq k \chi(S)$. Hence we have

$$
\chi(T(S))=\chi(A(S)) \leq k \chi(S)
$$


for all bounded sets $S$ in $\bar{U}$. Also $T(\bar{U})$ is bounded. Now the desired conclusion follows by an application of Theorem 2.3 to the multi-valued operator $T$ on $\bar{U}$. This completes the proof.

Corollary 3.2. Let $\mathcal{B}_{r}(0)$ and $\overline{\mathcal{B}_{r}(0)}$ denote respectively the open and closed balls in a Banach space $X$ centered at origin 0 of radius $r$. Let $A: \overline{\mathcal{B}_{r}(0)} \rightarrow \mathcal{P}_{c l, c v, b d}(X)$, $B: \overline{\mathcal{B}_{r}(0)} \rightarrow \mathcal{P}_{c p, c v}(X)$ two multi-valued operators satisfying

(a) $A$ is a multi-valued contraction with the contraction constant $k$, and

(b) $B$ is u.s.c. and compact.

Then either

(i) the operator inclusion $\lambda x \in A x+B x$ has a solution in $\overline{\mathcal{B}_{r}(0)}$ for $\lambda=1$, or

(ii) there is an element $u \in X$ with $\|u\|=r$ such that $\lambda u \in A u+B u$ for some $\lambda>1$.

Proof. We just show that $T\left(\overline{\mathcal{B}_{r}(0)}\right)$ is bounded, where $T x=A x+B x, x \in \mathcal{B}_{r}(0)$. From the definition of $T$ it follows that $T\left(\overline{\mathcal{B}_{r}(0)}\right) \subset A\left(\overline{\mathcal{B}_{r}(0)}\right)+B\left(\overline{\mathcal{B}_{r}(0)}\right)$. Since $B$ is compact, $B\left(\overline{\mathcal{B}_{r}(0)}\right)$ is a compact set and consequently a bounded subset of $X$. We only prove that $A\left(\overline{\mathcal{B}_{r}(0)}\right)$ is a bounded set in $X$. Let $x \in \overline{\mathcal{B}_{r}(0)}$ be arbitrary. Then for any $a \in A x$ we have

$$
\begin{aligned}
|a| & \leq\|A(x)\| \\
& \leq H(A(x), A(0))+\|A(0)\| \\
& \leq k|x|+\|A(0)\| \\
& \leq k r+\|A(0)\| \\
& <\infty,
\end{aligned}
$$

and so $A\left(\overline{\mathcal{B}_{r}(0)}\right)$ is bounded. Now the desired conclusion follows by an application of Theorem 2.3. This competes the proof.

The Kuratowskii measure $\alpha$ of noncompactness in a Banach space is a nonnegative real number $\alpha(S)$ defined by

$$
\alpha(S)=\inf \left\{r>0: S \subset \bigcup_{i=1}^{n} S_{i}, \text { and } \operatorname{diam}\left(S_{i}\right) \leq r, \forall i\right\}
$$

for all bounded subsets $S$ of $X$.

It is known that the Kuratowskii measure $\alpha$ of noncompactness has all the properties $\left(\chi_{1}\right)$ through $\left(\chi_{6}\right)$ of Hausdorff measure of noncompactness on $X$. The following useful results appear in Banas and Goebel [2].

It is known that the Kuratowskii measure $\alpha$ of noncompactness has all the properties $\left(\chi_{1}\right)$ through $\left(\chi_{6}\right)$ of Hausdorff measure of noncompactness on $X$. The following useful results appear in Banas and Goebel [2].

Lemma 3.5.([5, page 7]) Let $\alpha$ and $\chi$ denote respectively the Kuratowskii and Hausdorff measure of noncompactness in a Banach space $X$. Then $\alpha(S) \leq 2 \chi(S)$ for any bounded set $S$ in $X$. 
Lemma 3.6. If $A: X \rightarrow X$ is a single-valued Lipschitz map with a Lipschitz constant $k$, that is,

$$
\|A x-A y\| \leq k\|x-y\|
$$

for all $x, y \in X$, and for some real number $k>0$, then we have $\alpha(A(S)) \leq k \alpha(S)$ for any bounded subset $S$ of $X$.

Theorem 3.4. Let $U$ and $\bar{U}$ be respectively the open and closed subsets of a Banach space $X$ such that $0 \in U$. Let $A: \bar{U} \rightarrow X$ be a single-valued and $B: \bar{U} \rightarrow \mathcal{P}_{c p, c v}(X)$ be a multi-valued operator such that $A(\bar{U})+B(\bar{U})$ is bounded. Suppose that

(a) $A$ is contraction with a contraction $k<1 / 2$, and

(b) $B$ is u.s.c. and compact.

Then either

(i) the operator inclusion $\lambda x \in A x+B x$ has a solution for $\lambda=1$, or

(ii) there is an element $u \in \partial U$ such that $\lambda u \in A u+B u$ for some $\lambda>1$, where $\partial U$ is the boundary of $U$.

Proof. Since $A$ is single-valued contraction, it is continuous on $X$. Therefore the multi-valued operator $T: X \rightarrow \mathcal{P}_{p}(X)$ defined by $T(x)=(A+B)(x)=A x+B x$ is upper semi-continuous on $X$. Obviously $A: \bar{U} \rightarrow \mathcal{P}_{c p, c v}(X)$, since $\alpha(A x) \leq k \alpha(\{x\})=0$. As a result, we have $T: \bar{U} \rightarrow \mathcal{P}_{c p, c v}(X)$. Let $S$ be a bounded subset of $\bar{U}$. As $B$ is completely continuous, we have that $\chi(B(S))=0$. From Lemmas 3.5 and 3.6, and property $\left(\chi_{6}\right)$, it follows that

$$
\begin{aligned}
\chi(T(S)) & \leq \chi(A(S)+B(S)) \\
& \leq \leq \chi(A(S))+\chi(B(S)) \\
& \leq \alpha(A(S)) \\
& \leq k \alpha(S) \\
& \leq 2 k \chi(S) \\
& <\chi(S)
\end{aligned}
$$

whenever $\chi(S)>0$. Hence we have $\chi(T(S))<\chi(S), \chi(S)>0$ for all bounded sets $S$ in $\bar{U}$. Also $T(\bar{U})$ is bounded. Now the desired conclusion follows by an application of Theorem 2.3 to the multi-valued operator $T$ on $\bar{U}$. This completes the proof.

Corollary 3.3. Let $\mathcal{B}_{r}(0)$ and $\overline{\mathcal{B}_{r}(0)}$ denote respectively the open and closed balls in a Banach space $X$ centered at origin 0 of radius $r$. Let $A: \overline{\mathcal{B}_{r}(0)} \rightarrow X, B: \overline{\mathcal{B}_{r}(0)} \rightarrow$ $\mathcal{P}_{c p, c v}(X)$ two multi-valued operators satisfying

(a) A is a single-valued contraction with contraction constant $k<1 / 2$,

(b) $B$ is u.s.c. and compact.

Then either

(i) the operator inclusion $\lambda x \in A x+B x$ has a solution in $\overline{\mathcal{B}_{r}(0)}$ for $\lambda=1$, or

(ii) there is an element $u \in X$ with $\|u\|=r$ such that $\lambda u \in A u+B u$ for some $\lambda>1$. 
In the following section we will prove an existence theorem for a certain functional integral inclusion of mixed type by an application of the abstract fixed point theorem of this section.

\section{Functional Integral Inclusions}

Let $\mathbb{R}$ denote the real line. Given a closed and bounded interval $J=[0,1]$ in $\mathbb{R}$, consider the following functional integral inclusion (in short FII),

$$
x(t) \in q(t)+\int_{0}^{\mu(t)} k_{1}(t, s) F(s, x(\theta(s))) d s+\int_{0}^{\sigma(t)} k_{2}(t, s) G(s, x(\eta(s))) d s, \quad t \in J,
$$

where $q: J \rightarrow \mathbb{R}, k_{1}, k_{2}: J \times J \rightarrow \mathbb{R}, F, G: J \times \mathbb{R} \rightarrow \mathcal{P}_{p}(\mathbb{R})$ and $\mu, \theta, \sigma, \eta: J \rightarrow J$.

By a solution of the FII (4.1) we mean a function $x \in C(J, \mathbb{R})$ that satisfies

$$
x(t)=q(t)+\int_{0}^{\mu(t)} k_{1}(t, s) v_{1}(s) d s+\int_{0}^{\sigma(t)} k_{2}(t, s) v_{2}(s) d s,
$$

for some $v_{1}, v_{2} \in L^{1}(J, \mathbb{R})$ satisfying $v_{1}(t) \in F(s, x(\theta(s)))$ and $v_{2}(t) \in G(s, x(\eta(s)))$ a.e. for $t \in J$.

We also need the following definitions in the sequel.

Definition 4.1. A multi-valued map $F: J \rightarrow \mathcal{P}_{p}(\mathbb{R})$ is said to be measurable if for any $y \in \mathbb{R}$, the function $t \rightarrow d(y, F(t))=\inf \{|y-x|: x \in F(t)\}$ is measurable.

Definition 4.2. A measurable multi-valued function $F: J \rightarrow \mathcal{P}_{p}(\mathbb{R})$ is said to be integrably bounded if there exists a function $h \in L^{1}(J, \mathbb{R})$ such that $\|v\| \leq h(t)$ a.e. $t \in J$ for all $v \in F(t)$.

Remark 4.1. It is known that if $F: J \rightarrow \mathcal{P}_{c p}(\mathbb{R})$ is a an integrably bounded multivalued function, then the set $S_{F}^{1}$ of all Lebesgue integrable selections of $F$ is closed and non-empty. See Hu and Papageorgiou [10].

Denote

$$
\begin{gathered}
A \pm B=\{a \pm b \mid a \in A \text { and } b \in B\} \\
\lambda A=\{\lambda a \mid a \in A\} \\
|F(t, x)|=\{|u|: u \in F(t, x)\}
\end{gathered}
$$

and

$$
\|F(t, x)\|=\sup \{|u|: u \in F(t, x)\} .
$$

Definition 4.3. Let $E$ be a Banach space with norm $\|\cdot\|$. A multi-valued function $\beta: J \times E \rightarrow \mathcal{P}_{c l, b d}(E)$ is called Carathéodory if

(i) $t \rightarrow \beta(t, x)$ is measurable for each $x \in E$, and 
(ii) $x \rightarrow \beta(t, x)$ is an upper semi-continuous almost everywhere for $t \in J$.

Definition 4.4. A Carathéodory multi-valued function $\beta: J \times E \rightarrow E$ is called $L^{1}$-Carathéodory if for every real number $r>0$ there exists a function $h_{r} \in L^{1}(J, \mathbb{R})$ such that

$$
\|F(t, x)\|=\sup \{\|u\|: u \in F(t, x)\} \leq h_{r}(t) \quad \text { a.e. } t \in J
$$

for all $x \in E$ with $|x| \leq r$.

Denote

$$
S_{\beta}^{1}(x)=\left\{v \in L^{1}(J, \mathbb{R}) \mid v(t) \in \beta(t, x(t)) \text { a.e. } t \in J\right\} .
$$

Then we have the following lemmas due to Lasota and Opial [13].

Lemma 4.1. Let $E$ be a Banach space. If $\operatorname{dim}(E)<\infty$ and $\beta: J \times E \rightarrow \mathcal{P}_{b d, c l}(E)$ is $L^{1}$-Carathéodory, then $S_{\beta}^{1}(x) \neq \emptyset$ for each $x \in E$.

Lemma 4.2. Let $E$ be a Banach space, $\beta$ a Carathéodory multi-valued map with $S_{\beta}^{1} \neq \emptyset$ and let $\mathcal{L}: L^{1}(J, E) \rightarrow C(J, E)$ be a continuous linear mapping. Then the operator

$$
\mathcal{L} \circ S_{\beta}^{1}: C(J, E) \rightarrow \mathcal{P}_{b d, c l}(C(J, E))
$$

is a closed graph operator on $C(J, E) \times C(J, E)$.

In the sequel, let us denote

$$
S_{F}^{1}(x)=\left\{v \in L^{1}(J, \mathbb{R}) \mid v(t) \in \beta(t, x(\theta(t))) \text { a.e. } t \in J\right\} .
$$

and

$$
S_{G}^{1}(x)=\left\{v \in L^{1}(J, \mathbb{R}) \mid v(t) \in \beta(t, x(\eta(t))) \text { a.e. } t \in J\right\} .
$$

We consider the following hypotheses in the sequel.

$\left(H_{0}\right)$ The functions $\mu, \theta, \sigma, \eta: J \rightarrow J$ are continuous.

$\left(H_{1}\right)$ The function $q: J \rightarrow \mathbb{R}$ is continuous.

$\left(H_{2}\right)$ The functions $k_{1}, k_{2}$ are continuous on $J \times J$ with $K_{1}=\max _{t, s \in J}\left|k_{1}(t, s)\right|$ and $K_{2}=\max _{t, s \in J}\left|k_{2}(t, s)\right|$.

$\left(H_{3}\right)$ The multi-valued function $t \mapsto F(t, x)$ is integrably bounded for all $x \in \mathbb{R}$.

$\left(H_{4}\right)$ There exists a function $\ell \in L^{1}(J, \mathbb{R})$ such that

$$
H(F(t, x), F(t, y)) \leq \ell(t)|x-y| \quad \text { a.e. } \quad t \in J
$$

for all $x, y \in \mathbb{R}$.

$\left(H_{5}\right)$ The multi-valued function $G: J \times \mathbb{R} \rightarrow \mathcal{P}_{c p, c v}(\mathbb{R})$ is $L^{1}$-Carathéodory.

$\left(H_{6}\right)$ There exists a function $\phi \in L^{1}(J, \mathbb{R})$ with $\phi(t)>0$ a.e. $t \in J$ and a nondecreasing function $\psi: \mathbb{R}^{+} \rightarrow(0, \infty)$ such that

$$
\|G(t, x)\| \leq \phi(t) \psi(|x|) \text { a.e. } t \in J
$$

for all $x \in \mathbb{R}$. 
Lemma 4.3. Suppose that the hypothesis $\left(H_{4}\right)$ holds. Then for any $a \in F(t, x)$,

$$
|a| \leq \ell(t)|x|+\|F(t, 0)\|, \quad t \in J
$$

for all $x \in \mathbb{R}$.

Proof. Let $x \in \mathbb{R}$ be arbitrary. Then by the triangle inequality,

$$
\begin{aligned}
\|F(t, x)\| & =H(F(t, x), 0) \\
& \leq H(F(t, x), F(t, 0))+H(F(t, 0), 0) \\
& \leq H(F(t, x), F(t, 0))+\|F(t, 0)\|,
\end{aligned}
$$

for all $t \in J$. Hence for any $a \in F(t, x)$,

$$
\begin{aligned}
|a| & \leq\|F(t, x)\| \\
& \leq H(F(t, x), F(t, 0))+\|F(t, 0)\| \\
& \leq \ell(t)|x|+\|F(t, 0)\|,
\end{aligned}
$$

for all $t \in J$. The proof of the lemma is complete.

Theorem 4.1. Assume that the hypotheses $\left(H_{0}\right)-\left(H_{6}\right)$ hold. Suppose that there is a real number $r>0$ such that

$$
r>\frac{K_{1} L+\|q\|+K_{2}\|\phi\|_{L^{1}} \psi(r)}{1-K_{1}\|\ell\|_{L^{1}}}, \quad K_{1}\|\ell\|_{L^{1}}<1
$$

where $L=\int_{0}^{1}\|F(s, 0)\| d s$. Then the FII (4.1) has a solution on $J$.

Proof. Let $X=C(J, \mathbb{R})$. Consider the open ball $\mathcal{B}_{r}(0)$ in $X$ centered at origin 0 of radius $r$, where the real number $r$ satisfies the inequality (4.2). Define two operators $A$ and $B$ on $\overline{\mathcal{B}}_{r}(0)$ by

$$
A x=\left\{u \in X \mid u(t)=q(t)+\int_{0}^{\mu(t)} k_{1}(t, s) v(s) d s, t \in J \text { and } v \in_{F}^{1}(x)\right\}
$$

and

$$
B x=\left\{u \in X \mid u(t)=\int_{0}^{\sigma(t)} k_{2}(t, s) v(s) d s, t \in J \text { and } v \in S_{G}^{1}(x)\right\} .
$$

Then the FII (4.1) is equivalent to the operator inclusion

$$
x(t) \in A x(t)+B x(t), t \in J .
$$

We will show that the multi-valued operators $A$ and $B$ satisfy all the conditions of Theorem 3.2. Clearly the operators $A$ and $B$ are well defined since $S_{F}^{1}(x) \neq \emptyset \neq S_{G}^{1}(x)$ for each $x \in X$. 
We first show that $A x$ is a closed, convex and bounded subset of $X$ for each $x \in X$. Let $\left\{u_{n}\right\}$ be a sequence in $A x$ converging to a point $u$. Then there is a sequence $\left\{v_{n}\right\} \subset S_{F}^{1}(x)$ such that

$$
u_{n}(t)=q(t)+\int_{0}^{\mu(t)} k_{1}(t, s) v_{n}(s) d s
$$

and $v_{n} \rightarrow v$. Since $F(t, x)$ is closed for each $(t, x) \in J \times \mathbb{R}$, we have $v \in S_{F}^{1}(x)$. As a result

$$
u(t)=q(t)+\int_{0}^{\mu(t)} k_{1}(t, s) v(s) d s \in A x(t) \forall t \in J .
$$

Hence $A$ has closed values on $X$.

Again let $u_{1}, u_{2} \in A x$. Then the are $v_{1}, v_{2} \in S_{F}^{1}(x)$ such that

and

$$
u_{1}(t)=q(t)+\int_{0}^{\mu(t)} k_{1}(t, s) v_{1}(s) d s t \in J
$$

$$
u_{2}(t)=q(t)+\int_{0}^{\mu(t)} k_{1}(t, s) v_{2}(s) d s t \in J
$$

Now for any $\gamma \in[0,1]$,

$$
\begin{aligned}
& \gamma u_{1}(t)+(1-\gamma) u_{2}(t) \\
= & \gamma\left[q(t)+\int_{0}^{\mu(t)} k_{1}(t, s) v_{1}(s) d s\right]+(1-\gamma)\left[q(t)+\int_{0}^{\mu(t)} k_{1}(t, s) v_{2}(s) d s\right] \\
= & q(t)+\int_{0}^{\mu(t)} k_{1}(t, s)\left[\gamma v_{1}(s)+(1-\gamma) v_{2}(s)\right] d s \\
= & q(t)+\int_{0}^{\mu(t)} k_{1}(t, s) v(s) d s
\end{aligned}
$$

where $v(t)=\gamma v_{1}(t)+(1-\gamma) v_{2}(s) \in F(t, x)$ for all $t \in J$. Hence $\gamma u_{1}+(1-\gamma) u_{2} \in A x$ and consequently $A x$ is convex for each $x \in X$.

To prove $A$ has bounded values, let $x \in X$ be arbitrary and let $y \in A x$. Then there is a $v \in S_{F}^{1}(x)$ such that

$$
y(t)=q(t)+\int_{0}^{\mu(t)} k_{1}(t, s) v(s) d s, t \in J
$$


By $\left(H_{1}\right)$,

$$
\begin{aligned}
|y(t)| & \leq|q(t)|+\int_{0}^{\mu(t)}\left|k_{1}(t, s) \| v(s)\right| d s \\
& \leq|q(t)|+K_{1} \int_{0}^{1}|v(s)| d s \\
& \leq\|q\|+K_{1} \int_{0}^{1}\|F(s, x(\theta(t)))\| d s \\
& \leq\|q\|+K_{1} \int_{0}^{1} h(s) d s \\
& \leq\|q\|+K_{1}\|h\|_{L^{1}} .
\end{aligned}
$$

for all $t \in J$. Taking the supremum over $t$,

$$
\|y\| \leq\|q\|+K_{1}\|h\|_{L^{1}}
$$

for all $y \in T x$. Hence $A x$ is a bounded subset of $X$ for each $x \in X$. As a result, $A$ defines a multi-valued operator $A: X \rightarrow \mathcal{P}_{c l, c v, b d}(X)$.

Next we show that $B$ has compact values on $\overline{\mathcal{B}}_{r}(0)$. Now the operator $B$ is equivalent to the composition $\mathcal{L} \circ S_{G}^{1}$ of two operators on $L^{1}(J, \mathbb{R})$, where $\mathcal{L}: L^{1}(J, \mathbb{R}) \rightarrow X$ is a continuous operator defined by

$$
\mathcal{L} v(t)=\int_{0}^{\sigma(t)} k_{2}(t, s) v(s) d s
$$

To show $Q$ has compact values, it then suffices to prove that the composition operator $\mathcal{L} \circ S_{G}^{1}$ has compact values on $\overline{\mathcal{B}}_{r}(0)$. Let $x \in \overline{\mathcal{B}}_{r}(0)$ be arbitrary and let $\left\{v_{n}\right\}$ be a sequence in $S_{G}^{1}(x)$. Then, by the definition of $S_{G}^{1}, v_{n}(t) \in G(t, x(\eta(t)))$ a. e. for $t \in J$. Since $G(t, x(\eta(t)))$ is compact, there is a convergent subsequence of $v_{n}(t)$ (for simplicity call it $v_{n}(t)$ itself) that converges in measure to some $v(t)$, where $v(t) \in G(t, x(\eta(t)))$ a.e. for $t \in J$. From the continuity of $\mathcal{L}$, it follows that $\mathcal{L} v_{n}(t) \rightarrow \mathcal{L} v(t)$ pointwise on $J$ as $n \rightarrow \infty$. In order to show that the convergence is uniform, we first show that $\left\{\mathcal{L} v_{n}\right\}$ is an equi-continuous sequence. Let $t, \tau \in J$; then

$$
\left|\mathcal{L} v_{n}(t)-\mathcal{L} v_{n}(\tau)\right| \leq\left|\int_{0}^{\sigma(t)} k_{2}(t, s) v_{n}(s) d s-\int_{0}^{\sigma(\tau)} k_{2}(\tau, s) v_{n}(s) d s\right|
$$


for some $v_{n} \in S_{G}^{1}(x)$. This further implies that

$$
\begin{aligned}
\mid \mathcal{L} v_{n}(t)- & \mathcal{L} v_{n}(\tau)|\leq| \int_{0}^{\sigma(t)} k_{2}(t, s) v_{n}(s) d s-\int_{0}^{\sigma(\tau)} k_{2}(\tau, s) v_{n}(s) d s \mid \\
\leq & \left|\int_{0}^{\sigma(t)} k_{2}(t, s) v_{n}(s) d s-\int_{0}^{\sigma(t)} k_{2}(\tau, s) v_{n}(s) d s\right| \\
& +\left|\int_{0}^{\sigma(t)} k_{2}(\tau, s) v_{n}(s) d s-\int_{0}^{\sigma(\tau)} k_{2}(\tau, s) v_{n}(s) d s\right| \\
\leq & \left|\int_{0}^{1}\right| k_{2}(t, s)-k_{2}(\tau, s)|| v_{n}(s)|d s| \\
& +\left|\int_{\sigma(\tau)}^{\sigma(t)}\right| k_{2}(\tau, s)|| v_{n}(s)|d s|
\end{aligned}
$$

Since $v_{n} \in L^{1}(J, \mathbb{R})$, the right hand side of (4.6) tends to 0 as $t \rightarrow \tau$. Hence, $\left\{\mathcal{L} v_{n}\right\}$ is equi-continuous, and an application of the Arzelá-Ascoli theorem implies that there is a uniformly convergent subsequence. We then have $\mathcal{L} v_{n_{j}} \rightarrow \mathcal{L} v \in\left(\mathcal{L} \circ S_{F}^{1}\right)(x)$ as $j \rightarrow \infty$, and so $\left(\mathcal{L} \circ S_{F}^{1}\right)(x)$ is compact. Thus we have $B: \overline{\mathcal{B}}_{r}(0) \rightarrow \mathcal{P}_{c p, c v}(X)$.

Next we show that $A$ is a multi-valued contraction on $X$. let $x, y \in X$ be and $u_{1} \in A(x)$. Then $u_{1} \in X$ and

$$
u_{1}(t)=q(t)+\int_{0}^{\mu(t)} k_{1}(t, s) v_{1}(s) d s
$$

for some $v_{1} \in S_{F}^{1}(x)$. Since

$$
H(F(t, x(t)), F(t, y(t)) \leq \ell(t)|x(t)-y(t)|,
$$

we obtain that there exists $w \in F(t, y(t))$ such that

$$
\left|v_{1}(t)-w\right| \leq \ell(t)|x(t)-y(t)|
$$

Thus the multi-valued operator $U$ defined by $U(t)=S_{F}^{1}(y)(t) \cap K(t)$, where

Next we show that $A$ is a multi-valued contraction on $X$. let $x, y \in X$ be and $u_{1} \in A(x)$. Then $u_{1} \in X$ and

$$
u_{1}(t)=q(t)+\int_{0}^{\mu(t)} k_{1}(t, s) v_{1}(s) d s
$$

for some $v_{1} \in S_{F}^{1}(x)$. Since

$$
H(F(t, x(t)), F(t, y(t)) \leq \ell(t)|x(t)-y(t)|,
$$

we obtain that there exists $w \in F(t, y(t))$ such that

$$
\left|v_{1}(t)-w\right| \leq \ell(t)|x(t)-y(t)|
$$


Thus the multi-valued operator $U$ defined by $U(t)=S_{F}^{1}(y)(t) \cap K(t)$, where

$$
K(t)=\left\{w|| v_{1}(t)-w|\leq \ell(t)| x(t)-y(t) \mid\right\}
$$

has nonempty values and is measurable. Let $v_{2}$ be a measurable selection for $U$ (which exists by Kuratowski-Ryll-Nardzewski'selection theorem. See [4]). Then $v_{2} \in F(t, y(t))$ and

Define

$$
\left|v_{1}(t)-v_{2}(t)\right| \leq \ell(t)|x(t)-y(t)| \text { for all } t \in J
$$

$$
u_{2}(t)=q(t)+\int_{0}^{\mu(t)} k_{1}(t, s) v_{2}(s) d s .
$$

It follows that $u_{2} \in A(y)$ and

$$
\begin{aligned}
\left|u_{1}(t)-u_{2}(t)\right| & \leq\left|\int_{0}^{\mu(t)} k_{1}(t, s) v_{1}(s) d s-\int_{0}^{\mu(t)} k_{1}(t, s) v_{2}(s) d s\right| \\
& \leq \int_{0}^{\mu(t)} k_{1}(t, s)\left|v_{1}(s)-v_{2}(s)\right| d s \\
& \leq \int_{0}^{\mu(t)} \ell(t) k_{1}(t, s)|x(s)-y(s)| d s \\
& \leq K_{1}\|\ell\|_{L^{1}}\|x-y\|
\end{aligned}
$$

Taking the supremum over $t$, we obtain

$$
\left\|u_{1}-u_{2}\right\| \leq K_{1}\|\ell\|_{L^{1}}\|x-y\| .
$$

From this and the analogous inequality obtained by interchanging the roles of $x$ and $y$ we get that

$$
H(A(x), A(y)) \leq K_{1}\|\ell\|_{L^{1}}\|x-y\|,
$$

for all $x, y \in X$. This shows that $A$ is a multi-valued contraction since $K_{1}\|\ell\|_{L^{1}}<1$.

Next we show that $B$ is completely continuous on $X$. Let $S$ be a subset of $\overline{\mathcal{B}_{r}(0)}$. Then $\|x\| \leq r$ for all $x \in S$. First we prove that $B$ is totally bounded on $X$. To do this, it is enough to prove that $B(S)$ is a uniformly bounded and equi-continuous set in $X$. To see this, let $u \in B x$ be arbitrary. Then there is a $v \in S_{G}^{1}(x)$ such that

$$
u(t)=\int_{0}^{\sigma(t)} k_{2}(t, s) v(s) d s .
$$

Hence

$$
\begin{aligned}
|u(t)| & \leq \int_{0}^{\sigma(t)}\left|k_{2}(t, s) \| v(s)\right| d s \\
& \leq \int_{0}^{\sigma(t)} K_{2}\|G(s, x(\eta(s)))\| d s \\
& \leq \int_{0}^{\sigma(t)} K_{2} h_{r}(s) d s \\
& =K_{2}\left\|h_{r}\right\|_{L^{1}}
\end{aligned}
$$


for all $x \in S$ and so $B(S)$ is a uniformly bounded set in $X$. Again let $t, \tau \in J$. Then for any $y \in B x$, one has

$$
|y(t)-y(\tau)|=\left|\int_{0}^{\sigma(t)} k_{2}(t, s) v(s) d s-\int_{0}^{\sigma(\tau)} k_{2}(\tau, s) v(s) d s\right|
$$

for some $v \in S_{G}^{1}(x)$. This further implies that

$$
\begin{aligned}
|y(t)-y(\tau)| \leq & \left|\int_{0}^{\sigma(t)} k_{2}(t, s) v(s) d s-\int_{0}^{\sigma(\tau)} k_{2}(\tau, s) v(s) d s\right| \\
\leq & \left|\int_{0}^{\sigma(t)} k_{2}(t, s) v(s) d s-\int_{0}^{\sigma(t)} k_{2}(\tau, s) v(s) d s\right| \\
& \quad+\left|\int_{0}^{\sigma(t)} k_{2}(\tau, s) v(s) d s-\int_{0}^{\sigma(\tau)} k_{2}(\tau, s) v(s) d s\right| \\
\leq & \left|\int_{0}^{\sigma(t)}\right| k_{2}(t, s)-k_{2}(\tau, s)|| v(s)|d s| \\
& +\left|\int_{\sigma(\tau)}^{\sigma(t)}\right| k_{2}(\tau, s)|| v(s)|d s| \\
\leq & \int_{0}^{1}\left|k_{2}(t, s)-k_{2}(\tau, s)\right|\|G(s, x(\eta(s)))\| d s \\
\leq & \int_{0}^{1}\left|k_{2}(t, s)-k_{2}(\tau, s)\right| h_{r}(s) d s+K_{1}|p(t)-p(\tau)|
\end{aligned}
$$

where $p(t)=\int_{0}^{\sigma(t)} h_{r}(s) d s$.

Since $\mu$ and $q$ are continuous on the compact interval $J$, they are uniformly continuous. Therefore, we have

$$
|y(t)-y(\tau)| \rightarrow 0 \text { as } n \rightarrow \infty
$$

Thus $B(S)$ is uniformly bounded and equi-continuous set in $X$. Hence $B x$ is compact by Arzela-Ascoli theorem.

Next we show that $B$ is a upper semi-continuous multi-valued mapping on $X$. Let $\left\{x_{n}\right\}$ be a sequence in $S$ such that $x_{n} \rightarrow x_{*}$. Let $\left\{y_{n}\right\}$ be a sequence such that $y_{n} \in B x_{n}$ and $y_{n} \rightarrow y_{*}$. We shall show that $y_{*} \in B x_{*}$. Since $y_{n} \in B x_{n}$, there exists a $v_{n} \in S_{G}^{1}\left(x_{n}\right)$ such that

$$
y_{n}(t)=\int_{0}^{\sigma(t)} k_{2}(t, s) v_{n}(s) d s, \quad t \in J
$$


We must prove that there is a $v_{*} \in S_{G}^{1}\left(x_{*}\right)$ such that

$$
y_{*}(t)=\int_{0}^{\sigma(t)} k_{2}(t, s) v_{*}(s) d s, \quad t \in J
$$

Consider the continuous linear operator $\mathcal{K}: L^{1}(J, \mathbb{R}) \rightarrow C(J, E)$ defined by

$$
\mathcal{K} y(t)=\int_{0}^{\sigma(t)} k_{2}(t, s) v(s) d s, \quad t \in J
$$

Now $\left\|y_{n}-y_{*}\right\| \rightarrow 0$ as $n \rightarrow 0$. From Lemma 4.2 , it follows that $\mathcal{K} \circ S_{G}^{1}$ is a closed graph operator. Also from the definition of $\mathcal{K}$, we have $y_{n} \in \mathcal{K} \circ S_{G}^{1}\left(x_{n}\right)$. Since $y_{n} \rightarrow y_{*}$, there is a point $v_{*} \in S_{G}^{1}\left(x_{*}\right)$ such that

$$
y_{*}(t)=\int_{0}^{\sigma(t)} k_{2}(t, s) v_{*}(s) d s, \quad t \in J .
$$

This shows that $B$ is a upper semi-continuous operator on $X$. Thus $B$ is an upper semicontinuous and totally bounded and hence completely continuous multi-valued operators on $X$.

Now the operators $A$ and $B$ satisfy all the conditions of Theorem 3.2 and hence an application of it yields that either the conclusion (i) or the conclusion (ii) holds. We show that the conclusion (ii) is not possible. Let $\lambda u \in A u+B u$, for some $\lambda>1$. Then there are $v_{1} \in S_{F}^{1}(u)$ and $v_{2} \in S_{G}^{1}(u)$ such that

$$
\lambda u(t)=q(t)+\int_{0}^{\mu(t)} k_{1}(t, s) v_{1}(s) d s+\int_{0}^{\sigma(t)} k_{2}(t, s) v_{2}(s) d s .
$$

By Lemma 4.3,

$$
\begin{aligned}
|u(t)| \leq & \lambda|u(t)| \leq q(t)+\int_{0}^{\mu(t)}\left|k_{1}(t, s)\right|\left|v_{1}(s)\right| d s+\int_{0}^{\sigma(t)}\left|k_{2}(t, s) \| v_{2}(s)\right| d s \\
\leq & q(t)+\int_{0}^{\mu(t)}\left|k_{1}(t, s)\right| \ell(s)|u(\theta(s))| d s \\
& +\int_{0}^{\mu(t)}\left|k_{1}(t, s)\right|\|F(t, 0)\| d s+\int_{0}^{\sigma(t)}\left|k_{2}(t, s)\right|\|G(s, u(\eta(s)))\| d s \\
\leq & L+\|q\|+\int_{0}^{\mu(t)} K_{1} \ell(s)|u(\theta(s))|+\int_{0}^{\sigma(t)} K_{2} \phi(s) \psi(|u(\eta(s))|) d s \\
\leq & K_{1} L+\|q\|+K_{1}\|\ell\|_{L^{1}}\|u\|+K_{2}\|\phi\|_{L^{1}} \psi(\|u\|) .
\end{aligned}
$$

Taking the supremum over $t$, we obtain

$$
\|u\| \leq K_{1} L+\|q\|+K_{1}\|\ell\|_{L^{1}}\|u\|+K_{2}\|\phi\|_{L^{1}} \psi(\|u\|)
$$


Substituting $\|u\|=r$ in the above inequality yields

$$
r \leq \frac{K_{1} L+\|q\|+K_{2}\|\phi\|_{L^{1}} \psi(r)}{1-K_{1}\|\ell\|_{L^{1}}}
$$

which is a contradiction to (4.2). Hence the conclusion (ii) of Theorem 3.2 does not hold and consequently the operator inclusion $x \in A x+B x$ has a solution in $\overline{B_{r}}(0)$. This further implies that the FII (4.1) has a solution on $J$. This completes the proof.

\section{Applications}

As an application of the integral equations, we consider the following nonlinear functional two point boundary valued problems (BVP) of ordinary differential inclusion,

$$
\left\{\begin{array}{l}
x^{\prime \prime}(t) \in F(t, x(\theta(t)))+G(t, x(\eta(t))) \text { a.e. } t \in J \\
x(0)=0=x(1)
\end{array}\right.
$$

and

$$
\left\{\begin{array}{l}
x^{\prime \prime}(t) \in F(t, x(\theta(t)))+G(t, x(\eta(t))) \text { a.e. } t \in J \\
x(0)=0=x^{\prime}(1)
\end{array}\right.
$$

where $F, G: J \times \mathbb{R} \rightarrow \mathcal{P}_{f}(\mathbb{R})$ and $\theta, \eta: J \rightarrow J$ are continuous.

By a solution of the FBVP (5.1) or (5.2) we mean a function $x \in A C^{1}(J, \mathbb{R})$ that satisfies

$$
x^{\prime \prime}(t)=v_{1}(t)+v_{2}(t), \text { for all } t \in J,
$$

for some $v_{1} v_{2} \in L^{1}(J, \mathbb{R})$ satisfying $v_{1}(t) \in F(t, x(\theta(t)))$ and $v_{2}(t) \in G(t, x(\eta(t)))$ almost everywhere for $t \in J$, where $A C^{1}(J, \mathbb{R})$ is the space of continuous real-valued functions whose first derivative exists and is absolutely continuous on $J$.

Theorem 5.1. Assume that the hypotheses $\left(H_{1}\right)-\left(H_{4}\right)$ hold. Suppose that there exists a real number $r>0$ such that

$$
r>\frac{L+\|\phi\|_{L^{1}} \psi(r)}{4-\|\ell\|_{L^{1}}}, \quad\|\ell\|_{L^{1}}<4
$$

where $L=\int_{0}^{1}\|F(s, 0)\| d s$. Then the FBVP (5.1) has a solution on $J$.

Proof. The FBVP (5.1) is equivalent to the FII

$$
x(t) \in \int_{0}^{1} k(t, s) F(s, x(\theta(s))) d s+\int_{0}^{1} k(t, s) G(s, x(\eta(s))) d s, t \in J,
$$

where $k(t, s)$ is the Green's function associated with the linear homogeneous BVP

$$
\left\{\begin{array}{l}
x^{\prime \prime}(t)=0 \text { a.e. } t \in J \\
x(0)=0=x(1)
\end{array}\right.
$$


It is known that the Green's function $G(t, s)$ satisfies the inequality

$$
|k(t, s)| \leq \frac{1}{4} .
$$

Now the desired conclusion follows by an application of Theorem 4.1 with $q(t)=0$, $\mu(t)=1, \sigma(t)=1$ for all $t \in J$ and $k_{1}(t, s)=k_{2}(t, s)=k(t, s)$ for all $t \in J$. The proof is complete.

Theorem 5.2. Assume that the hypotheses $\left(H_{1}\right)-\left(H_{4}\right)$ hold. Suppose that there exists a real number $r>0$ such that

$$
r>\frac{L+\|\phi\|_{L^{1}} \psi(r)}{1-\|\ell\|_{L^{1}}}, \quad\|\ell\|_{L^{1}}<1,
$$

where $L=\int_{0}^{1}\|F(s, 0)\| d s$. Then the FBVP (5.2) has a solution on $J$.

Proof. The FBVP (5.2) is equivalent to the FII

$$
x(t) \in \int_{0}^{1} H(t, s) F(s, x(\theta(s))) d s+\int_{0}^{1} H(t, s) G(s, x(\eta(s))) d s, t \in J,
$$

where $H(t, s)$ is the Green's function associated with the linear homogeneous BVP

$$
\left\{\begin{array}{l}
x^{\prime \prime}(t)=0 \text { a.e. } t \in J \\
x(0)=0=x^{\prime}(1) .
\end{array}\right.
$$

It is known that the Green's function $H(t, s)$ satisfies the inequality

$$
|H(t, s)| \leq 1 \text {. }
$$

Now the desired conclusion follows by an application of Theorem 4.1 with $q(t)=0, \mu(t)=$ $1, \sigma(t)=1$ for all $t \in J$ and $k_{1}(t, s)=k_{2}(t, s)=H(t, s)$ for all $t, s \in J$. The proof is complete.

\section{References}

[1] J. Appell, H. T. Nguyen and P. Zabreiko, Multivalued superposition operators in ideal spaces of vector spaces III, Indag. Msth. 3(1992), 1-9.

[2] J. Banas and K. Goebel, Measures of Noncompactness in Banach Spaces, Marcel Dekker Inc., New York, 1980.

[3] J. Aubin and A. Cellina, Differential Inclusions, Springer Verlag, 1984.

[4] H. Covitz and S. B. Nadler Jr., Multivalued contraction mappings in generalized metric spaces, Israel J. Math. 8(1970), 5-11.

[5] K. Deimling, Multivalued Differential Equations, W. de Gruyter, 1992.

[6] B. C. Dhage, On some variants of Schauder's fixed point principle and applications to nonlinear integral equations, J. Math. Phy. Sci. 25(1988), 603-611. 
[7] B. C. Dhage, Some fixed point theorems in ordered Banach spaces and applications, The Math. Student 63(3-4) (1992), 81-88.

[8] B. C. Dhage, Multi-valued mappings and fixed points I, Nonlinear Funct. Anal. \& Appl. 10 (2005), 359-378.

[9] A. Granas and J. Dugundji, Fixed Point Theory, Springer Verlag, 2003.

[10] S. Hu and N. S. Papageorgiou, Handbook of Multivalued Analysis , Vol. I: Theory, Kluwer Academic Publishers Dordrechet / Boston / London, 1997.

[11] M. Kisielewicz, Differential Inclusions and Optimal Control, Kluwer Acad. Publ., Dordrecht, 1991.

[12] M. A. Krasnoselskii, Topological Methods in the Theory of Nonlinear Integral Equations, Pergamon Press, 1964.

[13] A. Lasota, and Z. Opial, An application of the Kakutani-Ky Fan theorem in the theory of ordinary differential equations, Bull. Acad. Pol. Sci. Ser. Sci. Math. Astronom. Phys. 13 (1965), 781-786.

[14] T. C. Lim, On fixed point stability for set-valued contraction mappings with applications to generalized differential equations, J. Math. Anal. Appl. 110 (1985), 436-441.

[15] M. Martelli, A Rothe's type theore for noncompact acyclic-valued maps, Bull. Un. Mat. Ital. 4(1975), 70-76.

[16] A. Petruşel, A generalization of Krasnoselskii's fixed point theorem, Proc. Sci. Comm. Meeting of "Aurel Vlaicu" Univ. Arad, vol. 14A (1996), 109-112.

[17] A. Petruşel, Multivalued operators and continuous selections. The fixed points set, P.U.M.A. 9 (1998), 165-170.

[18] W. V. Petryshyn and P. M. Fitzpatrtik, A degree theory, fixed point theorems, and mappings theorems for multi-valued noncompact mappings, Trans. Amer. Math. Soc. 194 (1974), $1-25$.

[19] D. O'Regan, Fixed-point theory for the sum of two operators, Appl. Math. Lett. 9 (1996), $1-8$.

[20] D. O'Regan, New fixed point results for 1-set contractive set-valued maps, Computes Math. Appl. 35(1998), 27-34.

[21] I. A. Rus, Technique of the fixed point structures for multivalued mappings, Math. Japonica 38 (1993), 289-296.

[22] L. Rybinski, An application of the continuous selection theorem to the study of the fixed points of multivalued mappings, J. Math. Anal. Appl. 153 (1990), 391-396.

[23] D. H. Tan, Two fixed point theorems of Krasnoselskii type, Rev. Roumaine Math. Pures Appl. 32 (1987), 397-400.

[24] Z. E. Zeidler, Nonlinear Functional Analysis and Its Applications : Part I, Springer Verlag, 1985.

Kasubai, Gurukul Colony, Ahmedpur- 413515 Dist : Latur, Maharashtra, India.

E-mail: bcd20012001@yahoo.co.in 